\title{
On the Equivalence of Coalitional and Individual Strategy-Proofness Properties*
}

\author{
Michel Le Breton ${ }^{\dagger} \quad$ Vera Zaporozhets ${ }^{\ddagger}$
}

September 2007 (Revised May 2008)

\begin{abstract}
In this paper, we introduce a sufficient condition on the domain of admissible preferences of a social choice mechanism under which the properties of individual and coalitional strategyproofness are equivalent. Then, we illustrate the usefulness of this general result in the case where a fixed budget has to be allocated among several pure public goods.
\end{abstract}

${ }^{*}$ We would like to express our deepest gratitude to our referee for an extremely careful and detailed report. His/her challenging questions did force us to be more acute on the scope of validity and the limits of our result.

†Université de Toulouse 1, Gremaq and Idei, France (lebreton@cict.fr)

‡Université de Toulouse 1, Gremaq, France and Université Catholique de Louvain, Core, Belgium (vera_zaporozhets@yahoo.com). 


\section{Introduction}

In social choice theory, we expect any collective decision within a group of individuals to reflect the preferences of its members over the feasible social alternatives. Since any individual is allowed to express freely his/her preference, it is however necessary to specify which alternative will be selected for each conceivable profile of preferences. This mapping is called hereafter a social choice mechanism. From a normative viewpoint, the concept is well defined since, as long as preferences are the only individual characteristics which matter, the mechanism simply describes which alternative should be selected in any possible set of circumstances. From a positive viewpoint, the mapping is truly a composed mapping, as an institution is an object which can be far more complicated than a direct revelation mechanism. An institution is described by a set of rules leading, from the perspective of the analysis, to a normal form game. The key observation is that when we account for equilibrium behavior in the setting describing that institution, we end up with a set of social alternatives which only depends upon the profile of preferences. This means that under the presumption that this set is nonempty and does not contain several alternatives, we can look at the composed map (amalgating the institution and the equilibrium behavioral responses) as a social choice mechanism.

In the context of a social choice mechanism, the strategic choice of an agent consists in reporting his/her preferences over the alternatives. In this revelation game, like in any game, the ultimate effect of his/her choice will depend upon his report together with the reports of the other individuals. There is no reason to assume that individuals will report the truth : if an agent can secure a better alternative by announcing preferences different from the truth, he/she may do so. These misreports can lead to a collective decision which has very little to do with the one based on true preferences and may turn out to be quite unsatisfactory. It becomes therefore important to identify which social choice mechanisms are immune to such manipulations.

In this paper, we will focus on two notions describing the resistance of a social choice mechanism to manipulations. The first one is strategyproofness which is a very strong form of robustness against "misbehavior". A social choice mechanism is strategyproof or individual strategyproof ( when we want to call the attention on the fact that only the behavior of individuals is taken into consideration) if telling the truth is a dominant strategy for every individual. This means that an individual does not need to solve the strategic uncertainty (attached, in principle to any game) to know what is his/her best strategy : no matter what the others do, a lie never pays out. This strong form of incentive compatibility is attractive 
but very demanding. In fact, an extremely dissapointing but fundamental result due to Gibbard (1973) and Satterthwaite (1975) states that if any preference can be reported, then only dictatorial mechanisms are strategyproof. In this paper, we are going to investigate the implication of strategyproofness for a class of environments where not every conceivable preference can be reported by an individual. We assume that an individual can report a preference from a prescribed subset of the set of all preferences, called the set of admissible preferences. Under that assumption of a restricted domain of preferences, the nihilist conclusion of the Gibbard-Satterthwaite impossibility theorem may disappear in the sense that there exist non dictatorial strategyproof social choice mechanisms. In this paper, we consider this general setting : for some domain of admissible preferences, strategyproofness leads to a very narrow class of mechanisms while for some others, the class may contain very satisfactory mechanisms. We dont touch the difficult and open question of characterizing the class of admissible domains of preferences leading to non dictatorial strategyproof social choice mechanisms.

The second notion of resistance to manipulation that we consider aims to incorporate the idea that besides individuals, groups (coalitions) of individuals may also play an active role, not captured by the notion of strategyproofness. Precisely, we want to consider a notion where the threats of coalitions are described and taken into consideration when designing the social choice mechanism. The definition of coalitional strategyproofness that we consider in this paper is the following. A social choice mechanism is coalitionally manipulable if for some profile of preferences, there exist a coalition such that when their members jointly misreport adequately their preferences, the mechanism selects an alternative that they all prefer to the one that would result if all of them had reported their true preferences.A social choice mechanism is coalitional strategyproof if it is never coalitionally manipulable. This definition is the conventional ${ }^{1}$ definition of coalitional strategyproofness used in the literature.

The main purpose of this paper is to study under which conditions the properties of (individual) strategyproofness and coalitional strategyproofness coincides. Strictly speaking, if a social choice mechanism is coalitional strategyproof then it is strategyproof but the converse does not need to hold true. Our main contribution is to identify a sufficient condition on the domain of admissible preferences for this equivalence to hold true. We call rich domains the domains satisfying this condition. Precisely, we show (theorem 1) that under the richness condition at hand, strategyproofness is equivalent to modified strong positive association (Barbera and Peleg (1990)), a condition that does not discriminate between

\footnotetext{
${ }^{1}$ It comes often under different names : coalition, coalitionally and group strategyproofness are among the most spread.
} 
an individual and coalitional level. Now, by this equivalence, strategyproofness inherits this indistinguishability between an individual and coalitional level. After introducing the formal definition of richness, we examine through many different domains the exact nature of the requirements which are needed for this property to hold. In particular while some flexibility is needed, we also show that it is logically independent of the free triple property ${ }^{2}$. In the second part of the paper, we illustrate the interest of this result through the examination of a specific social environment where the decision is on the allocation of a fixed amount of ressources (say a budget) between several public expenditures. We revisit an important impossibility result due to Zhou (1991). The proof scheme suggested by theorem 1 goes as follow. First, we prove that the domain under scrutiny is rich. Therefore, from theorem1, the restriction of a strategyproof mechanism to any subdomain is coalitional strategyproof. We select a subdomain, and exploit the geometry of the Pareto set over that subdomain or (better) take advantage of what is known (existing results in the literature) to sort out the implications of strategyproofness over that subdomain. Finally, go back to the original domain through a top only property. Along thse lines we obtain an alternative proof of a theorem slightly weaker than Zhou'soriginal statement. We also show how to use this proof scheme to deal with other domains not considered and covered by Zhou.

\section{Related Literature}

This paper is at the intersection of two branches of the literature. On one hand, we study the role of the domain of admissible preferences on the properties of a social choice mechanism satisfying some other conditions. On the other hand, we are mostly interested by the definition and implications of coalitional incentive compatibility constraints in the design of a social choice mechanism.

We are not the first to pay attention to the role of the domain in the characterization of strategyproof social choice mechanisms. As the Gibbard-Satterthwaite's theorem makes use of the universal domain condition, it was natural to investigate the responsability of that assumption in the derivation of the result. It was also useful since (besides voting) most environments of interest entail restricted preferences. This literature is nicely surveyed in Barbera (2001) and Sprumont (1995). To the best of our knowledge, very few general principles have been established and the research has consisted mostly in the detailed study of the implications of strategyproofness in some classes of problems. It is worth mentionning few of these general results. Dasgupta, Hammond and Maskin (1979) have introduced a general domain richness condition that they use as a generalization of the universal domain condition in the formulation of many results in the theory of implementation. In a general

\footnotetext{
${ }^{2}$ This question was raised by our referee.
} 
class of allocation environments (covering the cases with private components), Fleurbaey and Maniquet (1997) have introduced the notion of strict monotonic closedness and demonstrate that for such domains, strategyproofness, non-bossiness ${ }^{3}$ and equal treatment of equals imply no-envy. For the same class of domains, Moulin (1993) demonstrates that coalitional strategyproofness and equal treatment of equals imply also no-envy. He also notes that coalition strategyproofness could be replaced by Maskin monotonicity which, as noted by Fleurbaey and Maniquet is a stronger requirement than strategyproofness and non-bossiness together, under the Dasgupta, Hammond and Maskin richness condition. These domain richness conditions are logically unrelated to our richness domain condition. Given two profiles satisfying some properties, these conditions ask for the existence of a third profile satisfying also some properties. In contrast, our condition asks that for any single profile satisfying some properties, there exists a second profile satisfying some properties.

The strong form of coalitional incentive compatibility considered here has been incorporated by many authors in axiomatic social choice. For instance, Moulin, in many (e.g. Moulin (1994), (1999)) of his works on axiomatic cost sharing, uses it as a key axiom. Of course, the question of coalitional incentive compatibility raises many challenging problems and have been formulated differently by other authors. Within the general theory of implementation, Maskin (1979) shows that the set of social choice mechanisms which can be implemented in strong Nash equilibrium is much smaller than the set of those which are simply Nash implementable. With the weaker concept of coalition-proof Nash equilibrium, Bernheim and Whinston (1987) derives similar conclusions. Based on a differential approach, Laffont and Maskin (1980) show the difficulty to conciliate strategyproofness and coalitional incentives. This line of research follows some early work by Green and Laffont (1979) emphasizing the impossibility of constructing Clarke-Groves mechanisms which are robust to the formation of coalitions. They assume ${ }^{4}$ that side payments are possible among the members of the coalition and that coalitions do not face informational issues. When coalitions are confronted themselves to the issue of elicitating information about preferences, we open the door to a class of new and difficult problems where the games of side contracting have to be properly defined. Cremer (1996) revisits the family of Clarke-Groves mechanisms from that perpective and obtain few positive results. Several contributions in the traditional Bayesian mechanism design approach have paid attention to coalitional considerations in different settings ranging from auctions to general organizations. Laffont and Martimort (1997) have

\footnotetext{
${ }^{3}$ This property was introduced by Satterthwaite and Sonnenschein (1981). We dont define it precisely : it amounts to require that if by changing his report, an individual does not change his allocation, then he does not change the allocation of somebody else.

${ }^{4}$ They call strongly coalition incentive compatible this class of Clarke-Groves mechanisms.
} 
characterized quite generally the class of social choice mechanisms which are immune to coalitional side contracting in this Bayesian setting.

\section{Definitions and Notations}

In this section, we present the class of social choice environments which we consider and we introduce the main definitions and notations which are going to be used throughout this paper.

We are interested in a situation where a society (group) described by a finite set $N=$ $\{1,2, \ldots, n\}$ of individuals must decide which alternative to select out of a set $X$ of feasible alternatives. Each individual $i \in N$ is described by his/her preference $R_{i}$ over $X$, which is assumed to be a complete preorder ${ }^{5}$. We will denote respectively by $P_{i}$ and $I_{i}$ the strict preference and the indifference relations induced by $R_{i}$. Sometimes, we will represent a preference $R_{i}$ by a utility function $U_{i}$, but the reader should keep in mind that, in our framework, two utility functions representing the same preference will always be considered as equivalent. A profile of preferences is a vector $\pi \equiv\left(R_{1}, R_{2}, \ldots ., R_{n}\right)$ describing the preferences of each individual in the society. If $\pi$ is a profile of preferences and $S \subseteq N$ is a nonempty subset of individuals, then $\pi_{S}$ denotes the subprofile $\left(R_{i}\right)_{i \in S}$; when $S=N \backslash\{i\}$ for some $i \in N$, we denote $\pi_{-i}$ for $\pi_{S}$. If $\pi$ and $\pi^{\prime}$ are two profiles of preferences and $S \subseteq N$, then $\pi^{\prime \prime} \equiv\left(\pi_{S}, \pi_{N \backslash S}^{\prime}\right)$ denotes the profile such that $\pi^{\prime \prime}(i)=\pi(i)$ if $i \in S$ and $\pi^{\prime \prime}(i)=\pi^{\prime}(i)$ if $i \notin S$.

Definition 1 Let $\Pi$ be a subset of profiles. A social choice mechanism with domain $\Pi$ is a mapping $C$ from $\Pi$ into $X$.

If $\Pi$ consists of all possibles profiles, the domain is said to be universal. Otherwise, it is said to be restricted. The notion of domain is central in our paper as the results are driven by assumptions which will be formulated on the domain. We will limit our investigation to Cartesian domains i.e. domains such that $\Pi=\prod_{i=1}^{n} D_{i}$ where for all $i \in N, D_{i}$ is a nonempty subset of complete preorders over $X$.

The social choice mechanism reflects the aspirations and properties that this society wants to take into account to proceed in selecting a social alternative. The input of such mechanism is a profile of preferences. This means that once we know the diversity of opinions in the society, conflicts but also areas of agreement, we have, in principle, everything needed to pick up a compromise. To operate, the mechanism needs this input, but in most cases,

\footnotetext{
${ }^{5} \mathrm{~A}$ preorder is a reflexive and transitive binary relation.
} 
this input is not known or verifiable with certainty by all members of the society. Confronted with this difficulty, we could then consider a broader class of social choice mechanisms where the domain would be now a Cartesian set $M=\prod_{i=1}^{n} M_{i}$ where for all $i \in N, M_{i}$ is an abstract set of messages or reports that can be sent by individual $i$. As every individual is ultimately interested by the social alternative that will be selected, such a mechanism together with the profile $\pi$ of preferences generates a normal form game among the individuals which are assumed to be rational players : for each individual $i$, the choice of the message $m_{i}$ to be sent, constitutes a strategic choice.

Using the apparatus of game theory, we can predict the equilibrium behavior of the individuals and therefore the social outcome. In this paper, we focus on social choice mechanisms admitting equilibria in dominant strategies i.e. such that, for all profile $\pi$ in the domain $\Pi$, each individual $i$ has a dominant strategy. It is well known ${ }^{6}$ that for such equilibrium concept, there is no loss of generality in restricting attention to the class of social choice mechanisms introduced in definition 1 and to impose that the report of the truth is a dominant strategy for every individual in every possible circumstance.

Definition 2 A social choice mechanism $C$ with domain $\Pi$ is manipulable by individual $i$ at profile $\pi$ if there exists $R_{i}^{\prime} \in D_{i}$ such that $C\left(\pi_{-i}, R_{i}^{\prime}\right) P_{i} C(\pi)$. A social choice mechanism $C$ with domain $\Pi$ is strategyproof if there is no individual $i$ and no profile $\pi \in \Pi$ such that $C$ is manipulable by $i$ at $\pi$.

This property reflects the necessity to provide incentives to individuals to make sure that they report the right information. Strategyproofness is a strong form of incentive compatibility as it requires the existence of dominant strategies. From the perspective of constructing the social choice mechanism, this property acts as a constraint in the design of the rule.

Some few more definitions and notations are needed. From now on, we limit our attention ${ }^{7}$ to the case where $D_{i} \equiv D$ for all $i \in N .^{8}$ Let $D^{*} \equiv \cup_{x \in X} D_{x}$ where :

$$
D_{x} \equiv\{R \in D: x P y \text { for all } y \in X \backslash\{x\}\}
$$

\footnotetext{
${ }^{6}$ This is the so called revelation principle (Dasgupta, Hammond and Maskin (1979). The reader may consult Jackson (2001) for a nice overview of implementation theory.

${ }^{7}$ In what follows, we will often abusely use the expression domain for both $\Pi$ and $D$. Under this uniformity assumption, we dont see any risk of confusion.

${ }^{8}$ This assumption implies that there are no intrinsic ex ante differences among individuals and therefore rules out social environments with private dimensions. Many results of the paper can be extended to frameworks where this uniformity assumption is weakened. Note however that, in its current form, the modified strong positive association property cannot accomodate the structural indifferences attached to a domain with private components.
} 
$D_{x}$ is the set of preferences for which the alternative $x$ is uniquely best. Finally, let $X^{*} \equiv\left\{x \in X\right.$ such that $\left.D_{x} \neq \varnothing\right\}$ : an alternative $x$ is in $X^{*}$ if there exists an admissible preference with $x$ on top. Let $C^{*}$ be the restriction of $C$ to the subdomain $\Pi^{*} \equiv\left(D^{*}\right)^{n}$.

The following result will be used in some proofs. Let :

$$
C(\Pi) \equiv\{x \in X: x=C(\pi) \text { for some } \pi \in \Pi\}
$$

be the range of the mechanism $C$.

Lemma 1 Let $C$ be a strategyproof social choice mechanism with domain D. For all $\pi \in D^{n}$ and all $x \in C(\Pi)$, if $R_{i} \in D_{x}$ for all $i \in N$, then $C(\pi)=x$.

Definition $3 A$ social choice mechanism $C$ with domain $\Pi$ is regular if $C(\Pi) \subseteq X^{*}$.

To the best of our knowledge, this property is new. It requires that the range of the mechanism is contained in the subset of alternatives which appear on the top of an admissible preference. It is certainly controversial in any environment where an alternative which could be considered as a good social compromise is disregarded simply because at best, it appears on second position in any individual preference ${ }^{9}$. In most of this paper, we will consider environments where the property of regularity does not raise any problem. The following simple lemma will be useful.

Lemma 2 Let $C$ be a strategyproof and regular social choice mechanism with domain D. Then, $C(\Pi)=C^{*}\left(\Pi^{*}\right)$.

Proof : Since $C^{*}\left(\Pi^{*}\right) \subseteq C(\Pi)$, we are left to prove that $C(\Pi) \subseteq C^{*}\left(\Pi^{*}\right)$. Let $x \in C(\Pi)$. Since $C$ is regular, $x \in X^{*}$. Let $\pi \in D^{n}$ be such that $R_{i} \in D_{x}$ for all $i \in N$. By lemma 1 , $x=C(\pi)=C^{*}(\pi)$ and hence $x \in C^{*}(\Pi)$

The property described in the following definition has been introduced by Barbera et Peleg (1990).

Definition 4 A social choice mechanism $C$ with domain $D^{n}$ satisfies the modified strong positive association ${ }^{10}$ property if for all $\pi \in D^{n}$, all $i \in N$ and all $x \in C(\Pi)$, if $C(\pi)=x$ and $R_{i}^{\prime} \in D$ is such that $x P_{i}^{\prime} y$ for all $y \in C(\Pi) \backslash\{x\}$ such that $x R_{i} y$, then $x=C\left(\pi_{-i}, R_{i}^{\prime}\right)$.

The following lemma due to Barbera and Peleg will be useful.

Lemma 3 A strategyproof social choice mechanism $C$ with domain $\Pi$ satisfies the modified strong positive association property.

\footnotetext{
${ }^{9}$ Kim and Roush (1980) say that a domain satisfies condition FP (first place) if $X^{*}=X$.

${ }^{10}$ Muller and Satterthwaite (1977) have proved the equivalence of strategyproofness and strong positive association over the universal domain of complete orders. The modified positive association property is necessary but not sufficient in general for strategyproofness. Tanaka (2002) exhibits an association property which is both necessary and sufficient on the domain of continuous preferences.
} 
The notion of strategyproofness describes individual incentives to report the truth. The next notion deals with the behavior of coalitions ${ }^{11}$.

Definition 5 A social choice mechanism $C$ with domain $\Pi$ is manipulable by coalition $S$ at profile $\pi$ if there exists $\pi^{\prime} \in \Pi$ such that $C\left(\pi_{S}^{\prime}, \pi_{N \backslash S}\right) P_{i} C(\pi)$ for all $i \in S$. A social choice mechanism $C$ with domain $\Pi$ is coalitional strategyproof if there is no coalition $S$ and no profile $\pi \in \Pi$ such that $C$ is manipulable by $S$ at $\pi$.

Coalitional strategy proofness is obviously more demanding than strategyproofness. It requires that there are no profitable deviations from reporting the truth not only by individuals but also by groups of individuals. This property is rather demanding as it does not impose to the deviating coalition to be credible.

We conclude this section with an important notion that will be used in subsequent proofs and a technical lemma. Given a social choice mechanism $C$ with domain $D^{n}$, a profile $\pi \in D^{n}$ and a coalition $S \subseteq N$, we denote by $C_{S}\left[\pi_{N \backslash S}\right]$ the social choice mechanism defined over the subsociety $S$ with domain $D^{S}$ by :

$$
C_{S}\left[\pi_{N \backslash S}\right]\left(\pi_{S}^{\prime}\right)=C\left(\pi_{S}^{\prime}, \pi_{N \backslash S}\right) \text { for all } \pi_{S}^{\prime} \in D^{S}
$$

The range of the mechanism $C_{S}\left[\pi_{N \backslash S}\right]$ will be denoted $A_{S}\left[\pi_{N \backslash S}\right]$ : it describes the set of alternatives (options) attainable by coalition $S$ given the subprofile $\left[\pi_{N \backslash S}\right]$ of reports by individuals outside coalition $S$. These sets , called option sets by Barbera and Peleg (1990) ${ }^{12}$ will play a critical role in the rest of the paper. For all $i \in N$ and $\pi \in D^{n}$, the option set of coalition $\{i\}$ will be denoted $A_{i}\left[\pi_{-i}\right]$.

Lemma 4 Let $X$ be a metric space and $D$ be a subset of the set of continuous preferences over $X$. If $C$ is a strategyproof social choice mechanism with domain $D^{n}$ then for all $\pi \in D^{n}$ and all $S \subseteq N, A_{S}\left[\pi_{N \backslash S}\right] \cap X^{*}$ is a closed subset of $X^{*}$.

Proof : Let $\pi \in D^{n}, S \subseteq N$ and $x \in X^{*} \backslash A_{S}\left[\pi_{N \backslash S}\right]$. We claim that there exists $\varepsilon>0$ such that :

$$
B(x, \varepsilon) \cap A_{S}\left[\pi_{N \backslash S}\right]=\varnothing
$$

where denotes the open ball centered on $x$ with radius $\varepsilon$ i.e. $B(x, \varepsilon)=\{y \in X: d(x, y)<\varepsilon\}^{13}$. Suppose on the contrary that for all $\varepsilon>0$, there exists $z_{\varepsilon} \in B(x, \varepsilon) \cap X^{*}$ such that $z_{\varepsilon} \in$ $A_{S}\left[\pi_{N \backslash S}\right]$. Since $x \in X^{*}$, there exists $R^{*} \in D$ such that $R^{*} \in D_{x}$. Let $\pi^{\prime} \equiv\left(R^{*}, R^{*}, \ldots, R^{*}\right)$

\footnotetext{
${ }^{11}$ We call coalition any non empty subset of individuals.

${ }^{12}$ This technique has been pionnered by Laffond (1980).

${ }^{13} d$ denotes the distance attached to the metric space.
} 
$y \equiv C\left(\pi_{S}^{\prime}, \pi_{N \backslash S}\right)$. Since $y \neq x$ and $R^{*} \in D_{x}$ we deduce $: x P^{*} y$. Since preferences are continuous, we deduce that there exists $\delta>0$ such that for all $z$ in the ball $B(x, \delta): z P^{*} y$. Select such a $\varepsilon>0$ smaller than $\delta$ and $R^{\varepsilon} \in D$ such that such $R^{\varepsilon} \in D_{z_{\varepsilon}}$. Let $\pi^{\varepsilon} \equiv\left(R^{\varepsilon}, R^{\varepsilon}, \ldots, R^{\varepsilon}\right)$ and

$$
w=C\left(\pi_{S}^{\varepsilon}, \pi_{N \backslash S}\right)
$$

Since $z_{\varepsilon} \in A_{S}\left[\pi_{N \backslash S}\right]$, we deduce from lemma 1 that $w=z_{\varepsilon}$.

Without loss of generality, let $S \equiv\{1, \ldots ., s\}$ where $s \equiv \# S^{14}$ and consider the finite sequence of profiles $\left(\widetilde{\pi}^{j}\right)_{0 \leq j \leq s}$ defined as follows :

$$
\widetilde{R}_{i}^{j} \equiv\left\{\begin{array}{l}
R_{i} \text { for all } i \notin S \\
R^{\varepsilon} \text { for all } i \in\{1, \ldots \ldots, j\} \\
R^{*} \text { for all } i \in\{j+1, \ldots \ldots, s\}
\end{array}\right.
$$

Since $C$ is strategyproof, we deduce :

$$
C\left(\widetilde{\pi}_{S}^{j}, \pi_{N \backslash S}\right) R^{*} C\left(\widetilde{\pi}_{S}^{j+1}, \pi_{N \backslash S}\right) \text { for all } j=0, \ldots, s-1
$$

Since $C\left(\widetilde{\pi}_{S}^{0}, \pi_{N \backslash S}\right)=y$ and $C\left(\widetilde{\pi}_{S}^{s}, \pi_{N \backslash S}\right)=w=z_{\varepsilon}$, we deduce from above and transitivity of $R^{*}$ that $y R^{*} z_{\varepsilon}$, a contradiction to $z_{\varepsilon} P^{*} y$

\section{Rich Domains}

In the preceding section, we have introduced two notions of strategyproofness. Individual strategyproofness takes into consideration profitable dishonest reports by individuals while coalitional strategyproofness extends the requirement to all coalitional dishonest joint reports. As already pointed out, the two notions are nested : coalitional strategyproofness is more demanding than strategyprofness. It is not difficult to produce environments for which it is strictly more demanding. The purpose of this section is to identify a class of social environments for which the two notions coincide. Precisely, we introduce a condition on the domain $D$ of preferences which is sufficient for this equivalence to hold true. This class of domains, that we call rich domains hereafter, is defined as follows.

Definition 6 A domain $\Pi=D^{n}$ is rich if for all $R \in D$ and $x, y \in X$ such that $y P x$ and $y \in X^{*}$, there exists $R^{\prime} \in D$ such that $R^{\prime} \in D_{y}$ and for all $z \neq x$ such that $x R z$, we have $x P^{\prime} z$.

\footnotetext{
${ }^{14} \# S$ denotes the cardinality of the set $S$.
} 


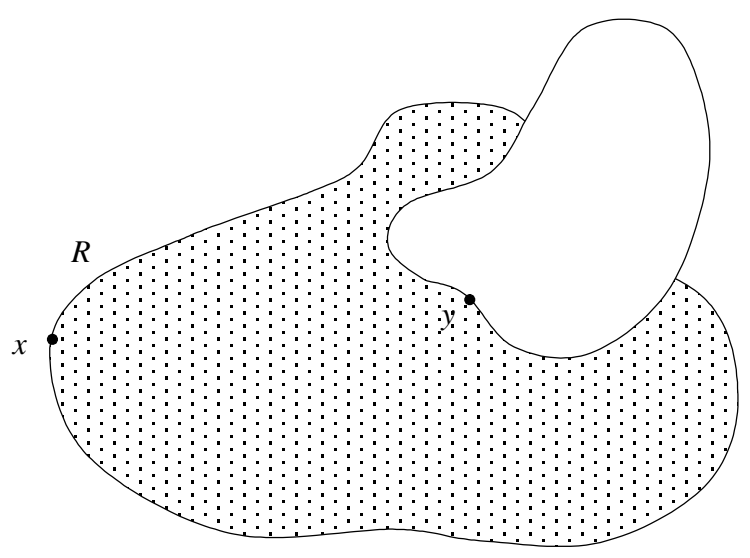

The notion of richness which is stated in definition 6 is designed to take advantage of the property of modified positive association. Without such domain condition, the property is vacuous as it cannot be used if it is never the case that two profiles are related as described by the premises of the condition.

To be rich, a domain must contain enough preferences. Of course, the universal domain is rich but there are also many restricted domains which meet this richness requirement. Intuitively, when a domain is rich we are able to consider transformations of individual preferences where the positions of two given alternatives are improved in the process. Precisely the alternative $y$ which was best among the two is now best among all and the other one $x$ still strictly dominates the alternatives that it was strictly dominating before but now $x$ also strictly dominates the alternatives belonging to its former indifference curve. This is illustrated on figures 1 and 2 in the case where alternatives are vectors in the two dimensional Euclidean space. On figure 1, we have drawn the upper contour sets of $x$ and $y$ for the preference $R$. On figure 2, we have reproduced the upper contour set of $x$ for $R$ and drawn, as a dotted curve, the upper countour set of $x$ for $R^{\prime}$. The upper contour set of $y$ for $R^{\prime}$ consists exclusively of $y$.

Figure 1 


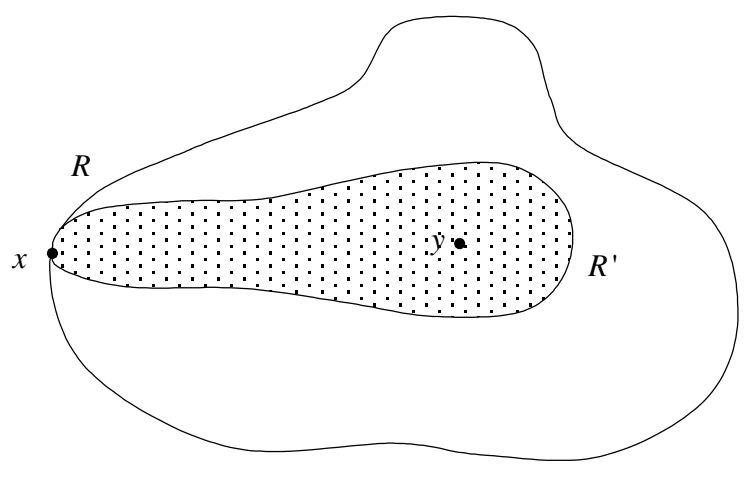

Figure 2

It should be transparent from this illustration that for a domain to be rich, we must have enough degrees of freedom to deform preferences. If not, the richness condition is likely to be violated. Consider for instance the traditionnal Euclidean environment popular in formal political science i.e. the setting where $X$ is some Euclidean space $\Re^{m}$ and $D$ is the subset of Euclidean preferences : a preference $R$ over $X$ is Euclidean if there exists $p \in \Re^{m}$ such that $x R y$ iff $\|x-p\| \leq\|y-p\|$. The upper contours sets are the spheres centered on $p$. The set of Euclidean preferences is not rich. To see why, consider the case where $m=2$. On figure 3 , we have drawn the upper contour set of $x$ with $y$ inside but different from $p$. As we can see immediately, necessarily, the circle centered on $y$ and containing $x$, has points outside the first disk.

\section{Figure 3}

We will see sevral important examples of rich domains in the next section. In addition to these environments, we can also prove ${ }^{15}$ that the set of continuous preferences over a metric

\footnotetext{
${ }^{15} \mathrm{~A}$ proof is available upon request from the authors.
} 


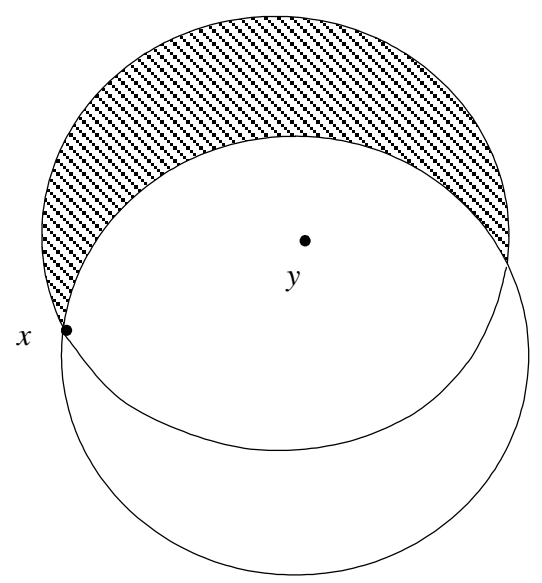

space considered by Barbera and Peleg (1990) is rich. Before illustrating richness through a sample of other domains, it is important to call the attention on the fact that there are general properties of preferences which preclude the richness condition. For instance, if $D$ is a subset of the set of separable preferences over a Cartesian set of alternatives ${ }^{16}$, then $D$ cannot be rich. To see why, consider the specific case ${ }^{17}$ where $X=\Re^{2}$ and $D$ is the subset of separable preferences with single peaked marginal preferences as defined by Barbera, Gul and Stachetti (1993) and Border and Jordan (1983). The domain $D$ contains preferences $R$ such that $p$ is best, $y P x$ and $x P z$ where the respective positions of $p, x$ and $y$ and $z$ are represented on figure 4 . The key features of this pattern are that $y$ does not belong to the rectangle generated by $x$ and $p$ and that $z$ belongs to the rectangle generated by $x$ and $y$. From the definition of $D$, it follows that any preference $R^{\prime} \in D$ such that $y$ is on top for $R^{\prime}$ implies that any alternatives $w$ in the rectangle generated by $x$ and $y$ is prefered to $\mathrm{x}$ according to $R^{\prime}$. In particular, we have $z R^{\prime} x$.

\section{Figure 4}

\footnotetext{
${ }^{16}$ Intuitively, a preference over a product space is separable if preferences over each factor of the product are defined without ambiguity. Such well defined projections are then called marginal preferences.

${ }^{17}$ A similar conclusion holds true for the domain of continuous and separable preferences considered by Le Breton and Weymark (1999).
} 


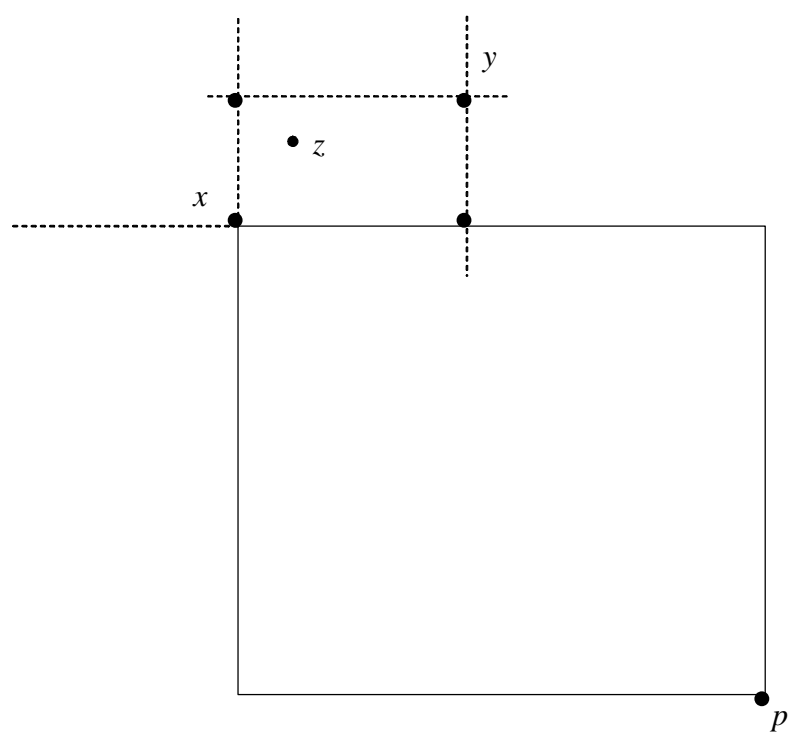

This list of examples shows that the richness condition calls for enough flexibility in order to distort a preference in the desired direction. Another illustration of that necessity is provided by the set $Q$ of quadratic preferences considered by Border and Jordan (1983) defined in the case where $X$ is some Euclidean space $\Re^{m}$. A preference $R$ is quadratic if there exists $p \in \Re^{m}$ and a symmetric and positive definite matrix $A=\left(a_{i j}\right)_{1 \leq i, j \leq m}$ such that $x R y$ iff $^{18}(x-p)^{t} A(x-p) \leq(y-p)^{t} A(y-p)$. The set of euclidean preferences considered earlier is a subset corresponding to the case where $\mathrm{A}$ is the identity matrix. Border and Jordan prove that when $D=Q$, if $C$ is strategyproof and onto, then $C$ is dictatorial. The set $D$ is not rich. Upper countour sets are now ellipses whose axis can be chosen arbitrarily but this gain in flexibility is not enough to obtain richness. For instance, if we consider a situation like the one depicted on figure 3, then any ellipse centered on $y$ and passing through $x$ will have the property that the symmetric image of $x$ through $y$ on the ellipse will be outside the original circle if $y$ is distant enough from $x$.

In evaluating the scope of validity of the richnesscondition, it is tempting to infer from the above examples that any "very small" domain of preferences is likely to violate the condition of richness; in these examples, preferences are described by a finite number of parameters and this is too much restrictive to reach the desired flexibility. Here is an example of domain

\footnotetext{
${ }^{18}$ For any $z \in \Re^{m}, z^{t}$ denotes the transpose of $z$.
} 
disproving this suspicion; in particular the domain has no free triples ${ }^{19}$. Consider the case where $X=\left\{x_{1}, x_{2}, \ldots, x_{m}\right\}$ is a finite set of $m$ points and let $D_{\text {cyclic }}$ be the domain consisting of the $m$ strict orders $x_{1} x_{2} \ldots x_{m}, x_{2} x_{3} \ldots x_{1}, \ldots, x_{m} x_{1} \ldots x_{m-1}$. This is a very small domain since a preference is fully described by its peak. It is however rich. Indeed, consider a preference $P$ in $D_{\text {cyclic }}$ and $x, y \in X$ such that $y P x$. We want to construct a preference $\widehat{P}$ with $y$ on top of $\widehat{P}$ and such that $x P z$ implies $x \widehat{P} z$. If $y$ is already in top of $P$ we are done. If not, then let $u$ be the top of $P$. Given the definition of $D_{\text {cyclic }}, y$ is somewhere betwwen $u$ and $x$ for the linear order $P$. If we lift up the entire linear order $P$ until $y$ reaches the top, we obtain a linear order $\widehat{P}$ where some of the alternatives which were ahead of $x$ are now below $x$ but all alternatives which were below $x$ are still below it for the new order. Kim and Roush (1980) have demonstrated that if a domain $D$ contains $D_{\text {cyclic }}$, then there exists a nondictatorial strategyproof social choice mechanism with domain $D^{n}$ iff there exists a nondictatorial strategyproof social choice mechanism with domain $D^{2}$. They provide also a beautiful characterization of the class of onto strategyproof social choice mechanisms over $D^{2}$. This family contains very nice nondictatorial mechanisms. To provide an intuitive description ot his class, it is useful to interpret the preferences in $D$ as the preferences of individuals for locations over the unit circle where, given his/her own location, an individual always prefer a location that is closer to his/her own when travelling always takes place in a clockwise direction around the circle (an one way road). They demonstrate that any 2person strategyproof mechanism $C$ is described by a list of integers $\left\{c_{j}\right\}_{1 \leq j \leq m}$ in $\{1,2, \ldots, m\}$ satisfying $c_{j} \leq c_{j+1}+1$ and $c_{m} \leq c_{1}+1$, such that :

$$
C\left(a_{i}, a_{j}\right)=\left\{\begin{array}{l}
a_{j} \text { if } i \leq j \leq i+c_{i}-1(\bmod m) \\
a_{i} \text { otherwise }
\end{array}\right.
$$

Kim and Roush also shows that if the domain $D_{\text {cyclic }}$ is enlarged to contain in addition the following $m$ strict orders $x_{1} x_{m} \ldots x_{2}, x_{2} x_{1} \ldots x_{3}, \ldots, x_{m} x_{m-1} \ldots x_{1}$, then any strategyproof social choice mechanism with a range containing at least three alternatives is dictatorial. By construction, the $m$ new preferences are obtained through the repeated application of the cyclic permutation to the preference $x_{1} x_{m} \ldots x_{2}$ instead of the preference $x_{1} x_{2} \ldots x_{m}$ used to for the construction of $D_{\text {cyclic }}$. Therefore we can use the same argument as above to conclude that this domain is rich. Finally, Kim and Roush have also been able to prove that for any integer $m$, there exist domains, on which every $k$-tuple is free, for $k=[\sqrt{\log m}]$ and admitting 2-person non dictatorial strategyproof and onto social choice mechanisms which always pick the peak of one of the two individuals. They offer a very elegant constructive

\footnotetext{
${ }^{19}$ This question was raised by our referee.
} 
proof of the domain but we have not examined whether their domain was rich or not. For the general finite setting, the derivation of a full characterization of the domains of linear orders for which the properties of strategyproofness and coalitional strategyproofness are equivalent appears to be a quite challenging combinatorial problem.

The interpretation of Kim and Roush's setting as describing the choice of the location of a facility on a circle opens the door to many alternative domains of preferences. One natural domain would consist of all single peaked preferences over the circle (when we can travel on this circular road clockwise or anticlockwise). Another domain, more restricted, would consist in preferences consistent with the distance on the circle. It is easy to show that the first domain is rich while the second is not. The argument behind the proof of these two claims is similar to the argument used in the case where the set of locations is segment instead of a circle. On the other hand, it is has been proved (see e.g. Schummer and Vohra (2002)) that in contrast to the case of a segment, strategyproofness over a domain of single peaked preferences over a circle leads to dictatoriality.

Finally, it is important to call the attention on the fact that in the premises of the definition of a rich domain, we only require the existence of this new "lifted" profile when $y \in X^{*}$. If $X \neq X^{*}$, this can make an important difference. To illustrate this point, consider the case where the set $X$ of alternatives is the unitary $m$-dimensional unitary simplex :

$$
\left\{x \in \Re_{+}^{m}: \sum_{k=1}^{m} x_{k}=1\right\}
$$

and $D$ is the set of linear preferences: a preference $R$ is linear if there exists a vector $a \in \Re^{m}$ such that $x R y$ iff $\langle a, x\rangle \geq\langle a, y\rangle$. In such a case, it is easy to show that $X^{*}$ is the set of vertices of the simplex. However, the domain is not rich as the definition of richness demands that if $x R z$ for some $z$, then $x P^{\prime} z$ for the new preference $R^{\prime}$. Given the linearity of indifference countour sets, it is not possible to alter weak preferences into strict preferences. This domain is important as it represents social choice mechanisms where chance is used in addition to individual preferences to select a social alternative: the set of alternatives $X$ is the set of lotteries on a set of $m$ pure alternatives. The property of linearity for individual preferences is equivalent to the requirement that preferences satisfy the von Neumann-Morgenstern axioms. In this setting, pionnered by Gibbard (1977) and further explored by Barbera (1979a) and others, a lot is known upon the class of strategyproof social choice mechanisms. Not all them are of course coalitional strategyproof. For instance, when $m=3$ and $n=2$, random dictatorship is not coalitional strategyproof : for instance, if vertices 1 and 2 are the peaks of the two individuals and vertex 3 is their common second 
choice, then reporting the truth is not an optimal strategy if their intensity for the second choice is large enough. Barbera (1979b) provides a description of the class of those which were coalitional strategy proof in that class.

Our main result on rich domains is the following.

Theorem 1 Let $C$ be a social choice mechanism with domain $\Pi=D^{n}$. If $D$ is rich, then $C$ is strategyproof if and only if $C$ is coalitional strategyproof.

Proof : Let $C$ be a strategyproof social choice mechanism on a domain $D^{n}$ assumed to be rich. We now prove that $C$ is coalitional strategyproof. assume on the contrary that $C$ is not coalitional strategyproof. Then, there exists $S \subseteq N$ and $\pi, \pi^{\prime} \in D^{n}$ such that for all $i \in S:$

$$
y \equiv C\left(\pi_{S}^{\prime}, \pi_{N \backslash S}\right) P_{i} C(\pi) \equiv x
$$

Since $D$ is rich, there exists $\pi^{\prime \prime} \in D^{n}$ such that for all $i \in S$ :

$$
R_{i}^{\prime \prime} \in D_{y} \text { and for all } z \neq x: x R_{i} z \Rightarrow x P_{i}^{\prime \prime} z
$$

and for all $i \notin S$ :

$$
R_{i}^{\prime \prime}=R_{i}
$$

Given the construction of $\pi^{\prime \prime}$ and since $C(\pi)=x$, a repeated application of lemma 3 leads to :

$$
C\left(\pi^{\prime \prime}\right)=x
$$

On the other hand, note that since $C$ is strategyproof, the restricted social choice mechanism $C_{S}\left[\pi_{N \backslash S}\right]=C_{S}\left[\pi_{N \backslash S}^{\prime \prime}\right]$ is also strategyproof. Since $x \in A_{S}\left[\pi_{N \backslash S}^{\prime \prime}\right]$ and $R_{i}^{\prime \prime} \in D_{y}$ for all $i \in S$, we deduce from lemma 1 that $C_{S}\left[\pi_{N \backslash S}^{\prime \prime}\right]\left(\pi_{S}^{\prime \prime}\right)=C\left(\pi^{\prime \prime}\right)=y$ in contradiction to $(1)$

While less important, there are also some other implications of the richness condition that we would like to report as they will be used as auxilliary results in the next section.

Definition 7 A social choice mechanism $C$ with domain $D^{n}$ is dictatorial if there exists an individual $i \in N$ such that for all $\pi \in D^{n}$ and all $x, y \in C(\Pi)$, if $x P_{i} y$, then $C(\pi) \neq y$.

A dictatorial social choice mechanism ignores the preferences of all but one individual : the most preferred alternative of this individual, called the dictator, is selected to be the social outcome. 
Lemma 5 Let $C$ be a regular and strategyproof social choice mechanism with domain $\Pi=D^{n}$. If $D$ is rich, then $C$ is dictatorial if and only $C^{*}$ is dictatorial.

Proof : Assume that $C^{*}$ is dictatorial and let us prove that $C$ is dictatorial too. Let $i$ be the dictator for $C^{*}$ and assume on the contrary that $i$ is not a dictator for $C$. Then there exist $\pi \in D^{n}$ and $x, y \in C(\Pi)$ such that $C(\pi)=x$ and $y P_{i} x$. Since $D$ is rich, there exists $R_{i}^{\prime} \in D$ such that :

$$
R_{i}^{\prime} \in D_{y} \text { and for all } z \neq x: x R_{i} z \Rightarrow x P_{i}^{\prime} z
$$

Further, since $C$ is regular, for all $j \in N \backslash\{i\}$, there exists $R_{j}^{\prime}$ such that :

$$
R_{j}^{\prime} \in D_{x}
$$

A repeated application of lemma 3 leads to $C\left(\pi^{\prime}\right)=x$. But, on the other hand, since $C\left(\pi^{\prime}\right)=C^{*}\left(\pi^{\prime}\right)$ and $i$ is a dictator for $C^{*}$, we deduce that $C\left(\pi^{\prime}\right)=y$ in contradiction to the earlier statement

\section{Applications}

The main purpose of this section is to illustrate the usefulness of theorem 1 though a detailed examination of a specific but important environment. When a domain of preferences $\Pi$ is rich, the analysis of the implications of strategyproofness in the construction of social choice mechanisms is considerably simplified as we know that the mechanism is in fact coalitional strategyproof. Note in particular that if a mechanism $C$ is coalitional strategyproof, then it is Pareto efficient over the range $C(\Pi)$ i.e. there does not exist $\pi \in \Pi$ and $x \in C(\Pi): x P_{i} C(\pi)$ for all $i \in N$.

Since Pareto Efficiency put some constraints on the subset of social outcomes that may be considered, this information can be exploited to simplify the analysis of the mechanism $C$.

\subsection{Allocation of a Budget Across Several Different Pure Public Goods}

The allocation environment considered in this section has been examined first by Zhou (1991) and is defined as follows. An exogeneous monetary budget of size normalized to 1 is to be 
allocated across $m$ different pure public goods. The set $X$ of alternatives is therefore the unitary $m$-dimensional unitary simplex :

$$
\left\{x \in \Re_{+}^{m}: \sum_{k=1}^{m} x_{k}=1\right\}
$$

We assume that each individual $i \in N$ has a preference over the $m$-dimensional positive orthant $\Re_{+}^{m}$ which is assumed to be strictly monotonic and strictly convex. The set $D$ is the set of restrictions of such preferences to the set $X$. It is straightforward to show that a preference $R$ is in $D$ iff its upper contour sets are strictly convex. Theorem 3 stated below holds true for all $n$ and all $m \geq 3$ but for the sake of simplicity, we will limit our investigation to the case where $m=3$ and $n=2$. The case where $m=2$ is considered in the next subsection.

Lemma 6 Let $m=3$ and $n=2$. Then the set $D$ of preferences with strictly convex upper contour sets is rich.

Proof : Let $R$ be a preference in $D$ such that $y P x$ for some $x, y \in X$. Let $A$ be the upper contour set of $x$ with respect to $R$ i.e.

$$
A=\{z \in X: z R x\}
$$

$A$ is a closed and strictly convex subset of $X$ with $y \in \operatorname{Interior} A$. Let $A^{\prime}$ be a closed and convex subset of $A$ such that $y \in$ Interior $A^{\prime}$ and Boundary $A \cap$ Boundary $A^{\prime}=\{x\}$. The construction of such subset is illustrated on figure $5^{20}$. The set $A^{\prime}$ as depicted is not strictly convex. To get a strict convex set $A^{\prime \prime}$ contained in $A^{\prime}$ and containing $y$, consider the line orthogonal to $\left[y^{\prime}, x\right]$ and passing through $\frac{x+y^{\prime}}{2}$. If we consider two circles centered respectively in $t$ and $u$, located on this line, on both sides of the segment $\left[y^{\prime}, x\right]$ and with radius $\|x-t\|=\|x-u\|$, then the intersection(s) of the circle(s) with either the half plane above or the half plane below the segment $\left[y^{\prime}, x\right]$ are contained in $A^{\prime}$ if $\|x-t\|$ is large enough. Since by construction this subset is strictly convex, the argument is complete. The construction of $A^{\prime \prime}$ is illustrated on figure 6 .

Let $J$ be the jauge of $\left(A^{\prime \prime}-\{y\}\right)$ with respect to $y$ i.e. the function defined by :

$$
J(w)=\underset{w-y \in \lambda\left(A^{\prime \prime}-\{y\}\right)}{\operatorname{Inf} \lambda}
$$

\footnotetext{
${ }^{20}$ The general existence of $A^{\prime}$ follows from the following argument. Since $y \in$ Interior $A$, there exists $\varepsilon>0$ such that $B(y, \varepsilon) \subset A$. The set $A^{\prime}$ defined as the convex hull of $B(y, \varepsilon)$ and $x$ satisfies the desired conditions.
} 


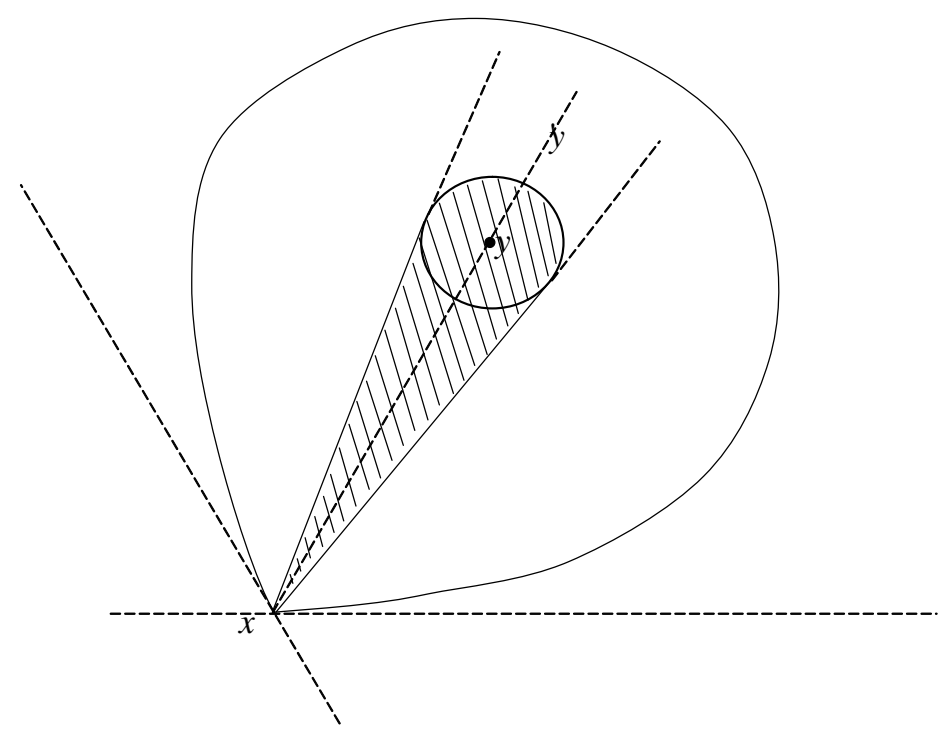

It is well known ${ }^{21}$ that $J$ is a continuous and convex (here strictly convex) function such that :

$$
J(w)=1 \text { iff } w \in \text { Boundary } A^{\prime \prime}
$$

Let $R^{\prime}$ be the preference generated by $-J$. By construction, $R^{\prime} \in D_{y}$ and for all $z \neq x$, $x R z$ implies $x P^{\prime} z$

\section{Figure 5}

\section{Figure 6}

Lemma 7 Let $m=3$ and $n=2$. Let $C$ be a strategyproof social choice mechanism over $D$ such that $C\left(D^{n}\right)=X$. Then, for all $x \in X$ and all $R_{1}, R_{1}^{\prime} \in D_{x}, A_{2}\left(R_{1}\right)=A_{2}\left(R_{1}^{\prime}\right)$.

Proof : Assume on the contrary that there exists $z \in A_{2}\left(R_{1}\right)$ such that $z \notin A_{2}\left(R_{1}^{\prime}\right)$. We construct a preference $R_{2}$ as follows. On one hand, since from lemma $4, A_{2}\left(R_{1}^{\prime}\right)$ is closed,

\footnotetext{
${ }^{21}$ See e.g. Rockafellar (1970).
} 


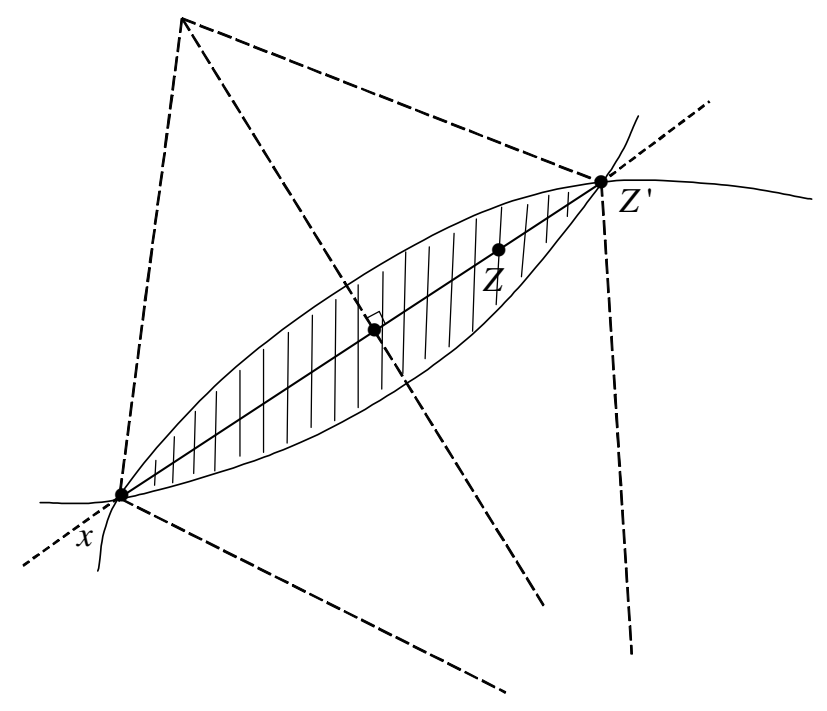

there exists a ball $B(z, \varepsilon)$ where $\varepsilon>0$ such that $B(z, \varepsilon) \cap A_{2}\left(R_{1}^{\prime}\right)=\varnothing$. On the other hand, from lemma 1 , we deduce that $x \in A_{2}\left(R_{1}^{\prime}\right)$. Let :

$$
w \equiv \text { Boundary } B(z, \varepsilon) \cap[x, z]^{22}
$$

Since $R_{1}$ is strictly convex : $w P_{1} z$. Since $R_{1}$ is continuous, we deduce therefore that there exists a ball $B(w, \delta)$ where $\frac{\varepsilon}{2}>\delta>0$ such that for all $u \in B(w, \delta): u P_{1} z$. Let $\left\{u^{\prime}, u^{\prime \prime}\right\} \equiv$ Boundary $B(z, \varepsilon) \cap$ Boundary $B(w, \delta)$. Consider the two half- lines with origin $x$ and going respectively through $u^{\prime}$ and $u^{\prime \prime}$ and the convex set $S$ as on figure $7^{23}$.

\section{Figure 7}

Proceeding as in the proof of lemma 6 , let $H$ be defined over $X$ as the jauge of $S$ with respect to $z$ and $R_{2}$ be the preference generated by $H$. We deduce that $R_{2}$ is strictly convex.

\footnotetext{
${ }^{23}$ As pointed out by the referee, the set $S$, as depicted, is not strictly convex. To get a strict convex set contained in $S$ and containing $z$, we can proceed as in the proof of lemma 6 . Consider the line orthogonal to $\left[z^{\prime}, x\right]$ and passing through $\frac{x+z^{\prime}}{2}$. If we consider a circle centered in a point $t$ located on this line and with radius $\|x-t\|$, then the intersection(s) of the circle(s) with either the half plane above or the half plane below the segment $\left[z^{\prime}, x\right]$ are contained in $S$. Since by construction this subset is strictly convex, the argument is complete.
} 


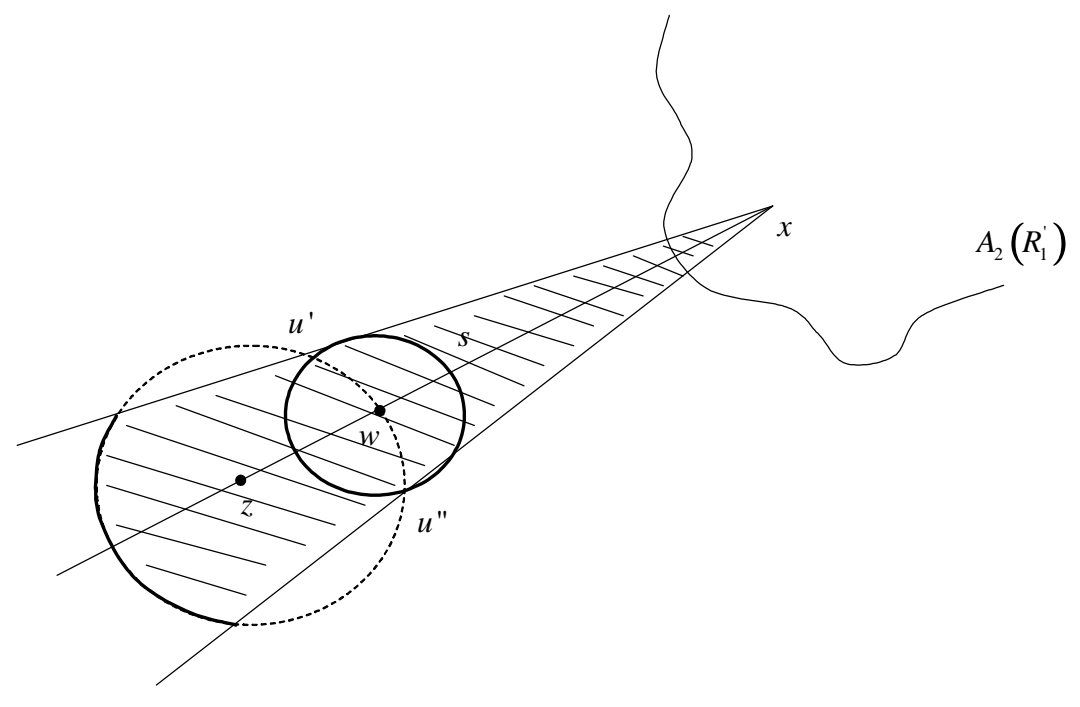

Further, $z$ is the unique best element and the boundary of $S$ is the indifference curve going through $x$.

Since, by assumption, $z \in A_{2}\left(R_{1}\right)$, there exists $R_{2}^{\prime} \in D$ such that $z=C\left(R_{1}, R_{2}^{\prime}\right)$. Since $C$ is strategyproof, we deduce therefore that :

$$
C\left(R_{1}, R_{2}\right)=z
$$

Now, let $B$ be the set ${ }^{24}$ of best alternatives of $R_{2}$ over $A_{2}\left(R_{1}^{\prime}\right)$. By construction of $B(z, \varepsilon)$, $B \cap B(z, \varepsilon)=\varnothing$. Also, by construction of $S$ and since $x \in A_{2}\left(R_{1}^{\prime}\right): B \subset S$. Further, since $C$ is strategyproof, we deduce therefore that there exist $b \in B$ such that:

$$
C\left(R_{1}^{\prime}, R_{2}\right)=b
$$

From the construction of $S$ and the position of $b$ in $S$, we deduce from the strict convexity of $R_{1}$ that $b P_{1} z$. Indeed, $b$ is necessarily such that $b=\lambda v+(1-\lambda) x$ for some $\lambda \in[0,1]$ and $v \in B(w, \delta)$. Therefore, since $v P_{1} z$ and $x P_{1} z$, we deduce from the strict convexity of $R_{1}$ that $b P_{1} z$. Comparing (2) and (3), this implies then that $C$ is manipulable by individ-

\footnotetext{
${ }^{24}$ Since the set $A_{2}\left(R_{1}^{\prime}\right)$ is compact, the set $B$ is nonempty. Note however, that,since $A_{2}\left(R_{1}^{\prime}\right)$ is not necessarily convex, the set $B$ may contain more than one alternative.
} 
ual 1 at the profile $\pi=\left(R_{1}, R_{2}\right)$ in contradiction to our assumption that $C$ is strategyproof

We are now in position to prove the main result of this section. To proceed, we will use a result proved by Bordes, Laffond and le Breton (1990) for the domain of Euclidean preferences over $X$. Let $\widehat{D}$ be the subset of Euclidean preferences over $\Re^{2}$ such that their ideal point belongs to $X$. Without any risk of confusion, we identify $\widehat{D}$ with $X$.

Theorem 2 Let $m=3$ and $n=2$. Let $C$ be a coalitional strategyproof social choice mechanism over $\widehat{D}$ such that $C\left(\widehat{D}^{n}\right)=X$. Then, $C$ is dictatorial.

Theorem 3 Let $m=3$ and $n=2$. Let $C$ be a strategyproof social choice mechanism over $D$ such that $C\left(D^{n}\right)=X$. Then, $C$ is dictatorial.

Proof : From lemma 7, $D$ is rich and therefore, from theorem $1, C$ is coalitional strategyproof. Let $\widehat{C}$ be the restriction of $\mathrm{C}$ to $\widehat{D}^{n}$. Then, $\widehat{C}$ is also coalitional strategyproof. We deduce from theorem 2 that $\widehat{C}$ is dictatorial. Without loss of generality, let individual 1 be the dictator for $\widehat{C}$. We now prove that 1 is also a dictator for $C$. This is equivalent to show that for all $\pi \in D^{2}$, the option set $A_{2}\left(R_{1}\right)$ is equal to the unique best element of $R_{1}$.

Let :

$$
\widehat{A}_{2}\left(R_{1}\right) \equiv\left\{x \in X: x=C\left(R_{1}, R_{2}\right) \text { for some } R_{2} \in \widehat{D}\right\}
$$

From lemma 3, we deduce that if $z=C\left(R_{1}, R_{2}\right)$, then $z=C\left(R_{1}, R_{2}^{\prime}\right)$ where $R_{2}^{\prime} \in \widehat{D}_{z}$. From lemma $6, \widehat{A}_{2}\left(R_{1}\right)=A_{2}\left(x_{1}\right)$ where $x_{1}$ denotes both the best alternative for $R_{1}$ and the Euclidean preference with ideal point $R_{1}$. By combining both claims, we obtain that :

$$
A_{2}\left(R_{1}\right)=A_{2}\left(x_{1}\right)=\widehat{A}_{2}\left(x_{1}\right)
$$

But, since 1 is a dictator for $C, \widehat{A}_{2}\left(x_{1}\right)=\left\{x_{1}\right\}$ and the conclusion follows $\square$

\subsection{Single Peakedness}

Theorem 3 was derived under the assumption that there are at least three different public goods. When there are only two public goods, the set $X$ is an interval. A preference in $D$ over that interval is single peaked. We know that for this social environment there are many non dictatorial strategyproof social choice mechanisms, on top of which the so called median mechanism. The general family of strategyproof social choice mechanisms has been characterized by Moulin (1980). The domain $D$ is rich and a shorter proof of the characterization result exploiting theorem 1 could be provided. But more importantly, this 
setting is interesting as it illustrates the fact that there are domains where individual and coalitional strategyproofness are equivalent without being equivalent to dictatoriality.

In fact, the richness property continues to hold in the case where single peakedness is defined with respect to an arbitrary tree instead of a segment, as in Demange (1982). The class of strategyproof social choice mechanisms operating over such a larger domain $D$ of singlepeaked preferences has been characterized by Danilov (1994). Since $D$ is rich, we deduce from theorem 1 that these mechanisms are coalitional strategyproof. A direct proof of that assertion is provided by Danilov.

\subsection{Piecewise Linear Preferences}

Theorem 3 can be extended to domains larger than $D$. Since here $X^{*}=X$, any social choice mechanism is trivially regular. From lemma 4 , we deduce that $C$ is dictatorial iff $C^{*}$ is dictatorial. Therefore, it is enough to prove that $C^{*}$ is dictatorial. To do so, it is useful to observe again that in the proof of lemma 6 , we do not exploit the full force of the strict convexity of $R_{1}$. What is truly needed is the strict monotonicity along any half-line with the best alternative $x_{1}$ as origin, a property called star-shapedness by Border and Jordan (1983).

The structure of the proof of theorem 3 is quite instructive. Once we know that the social choice mechanism $C$ is coalitional strategyproof, we can exploit the simple fact that any restriction of $C$ to a subdomain is also coalitional strategyproof. On these subdomains, the geometry of the Pareto set is sometimes easy to derive. For instance, in the case where the subdomain consists of the subset of Euclidean preferences, the Pareto set is the convex hull of the ideal points of the two individuals. The proof of theorem 2 based on the technique of option sets uses this property. Once we know what happens on a subdomain, it remains of course to extend the result to the all domain. The key step ${ }^{25}$, which corresponds here to lemma 7, is a "top only" property asserting that strategyproofness implies that only the top alternatives of the two individuals matter in calculating the social outcome.

Theorem 3 is a slightly weaker version of an impossibility result established for this environment by Zhou (1991). His setting is identical to the one considered here but instead of us, Zhou does not assume that the range of the mechanism $C$ coincides with $X$ and demonstrates his result under the weaker assumption that the range of $C$ is two dimensional.

To illustrate the usefulness of this method, we present a variant of the technique developed in the preceding section and derive a new result. Once again, after a proof that the domain we are going to consider is rich, it takes advantage of existing results on strategyproofness

\footnotetext{
${ }^{25}$ This "tops only" property is a familiar cornerstone in this area.
} 


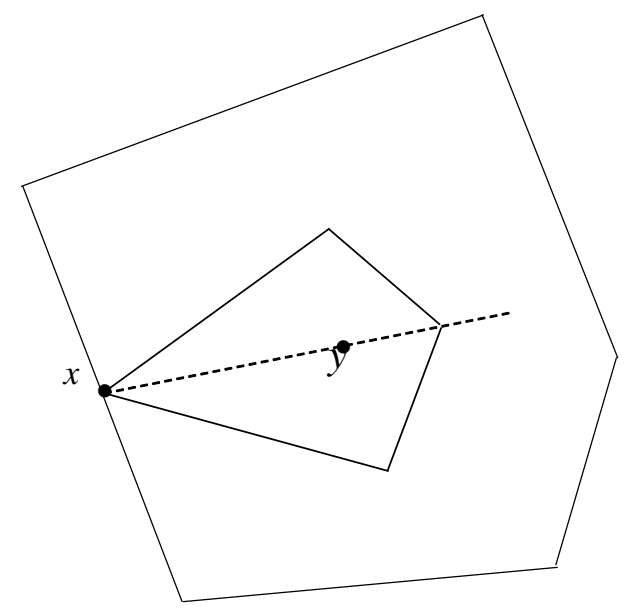

on a subdomain of that domain. Within the public good setting described in the preceding section, we consider now the set $D$ of piecewise linear preferences over $X$. A preference $R$ is piecewiselinear if there exists a finite set of vectors $a^{1}, a^{2}, \ldots ., a^{K} \in \Re^{m}$ such that for all $x, y \in X$ :

$$
x R y \text { iff } \operatorname{Min}\left(\left\langle a^{1}, x\right\rangle, \ldots .,\left\langle a^{K}, x\right\rangle\right) \geq \operatorname{Min}\left(\left\langle a^{1}, y\right\rangle, \ldots .,\left\langle a^{K}, y\right\rangle\right)
$$

It can be verified that the domain $D$ is rich. A proof similar to the proof used in the preceding section and based on the jauge function can be offered. An illustration of the argument behind this assertion is provided on figure 7 .

\section{Figure 7}

Let $C$ be strategyproof social choice mechanism over $D^{n}$. Since $D$ is rich, we deduce from theorem 1 that it is coalitional strategyproof. This implies that its restriction $\widehat{C}$ to the subdomain of linear preferences is also coalitional strategyproof. From the results of Barbera and Gibbard, to which we already alluded, we deduce that $\widehat{C}$ is dictatorial. It remains to prove that $C$ itself is dictatorial. To do so, it is enough to have a top only property similar to the one stated in lemma 7 in the case of the preceding domain. We leave to the reader 
to check that a statement analogous to lemma 7 holds true here: as already pointed out, a close look at the argument used in the proof shows that we do not exploit the full force of strict convexity.

We could produce much more results along the same lines. For instance, within the class of piecewise linear preferences, we could limit ourselves to the subclass of those for which $K$ (the number of linear forms in the definition of the preference) is bounded by some exogenous integer. This bound has of course implications on the shape of upper countour sets. It is interesting to observe that when $K=2$ and $m=3$, the set $X^{*}$ coincides with the boundary of the simplex. Once again the proof can be adjusted to deal with this domain which is a subdomain of the domain of convex preferences but a superdomain of the domain of linear preferences.

Much more illustrations could be provided. It is straightforward to see that the conclusion of theorem 3 holds true for the social environment where $X=\Re_{+}^{2}$ and $D$ is the set of preferences with compact and strictly convex upper contour sets. However, within this cartesian setting, this conclusion does not hold true when preferences are further restricted to be separablef. In fact, as demonstrated by Le Breton and Sen (1999) when a domain $D$ consists of separable preferences over a product set, strategyproofness implies decomposability. The class of decomposable strategyproof social choice mechanisms contains non dictatorial mechnisms. However, these mechanisms are not Pareto efficient. If we insist on Pareto efficiency, then the class of strategyproof social choice mechanisms collapses on dictatorial mechanisms.

\section{References}

[1] Aumann, R.J. 1959. "Acceptable Points in General Cooperative n-Person Games", in Contributions to the Theory of Games, Vol IV, Tucker, A.W. and R.D. Luce, eds., Princeton University Press, Princeton, 1959.

[2] Barbera, S. 1979a. "Majority and Positional Voting in a Probabilistic Framework", Review of Economic Studies, 46, 389-397.

[3] Barbera, S. 1979b. "A Note on Group Strategy-Proof Decision Schemes", Econometrica, 47, 637-640.

[4] Barbera, S. 2001. "An Introduction to Strategy-Proof Social Choice Functions", Social Choice and Welfare, 18, 619-653. 
[5] Barbera, S., Gul, F. and E. Stacchetti. 1993. "Generalized Median Voter Schemes and Committees", Journal of Economic Theory, 61, 262-289.

[6] Barbera, S. and B. Peleg. 1990. "Strategy-Proof Voting Schemes with Continuous Preferences", Social Choice and Welfare, 7, 31-38.

[7] Bernheim, D., Peleg, B. and M.D. Whinston 1987. "Coalition-Proof Nash Equilibria: I Concepts", Journal of Economic Theory 42, 1-12.

[8] Bernheim, D. and M.D. Whinston 1987. "Coalition-Proof Nash Equilibria: II Applications", Journal of Economic Theory 42, 13-29.

[9] Border, K. and J. Jordan. 1981. "Straightforward Elections, Unanimity and Phantom Voters", Review of Economic Studies, 50, 153-170.

[10] Bordes, G., Laffond, G. and M. Le Breton. 1990. "Strategy-Proofness Issues in Some Economic and Political Domains", Unpublished Manuscript, University of Bordeaux.

[11] Cremer, J. 1996. "Manipulation by Coalition under Asymmetric Information : The Case of Groves Mechanisms", Games and Economic Behavior, 13, 39-73.

[12] Danilov, V.I. 1994. "The Structure of Non-Manipulable Social Choice Rules on a Tree", Mathematical Social Sciences, 27, 123-131.

[13] Dasgupta, P., Hammond, P.J. and E. Maskin. 1979. "The Implementation of Social Choice Rules", Review of Economic Studies, 46, 185-216.

[14] Demange, G. 1982. "Single Peaked Orders on a Tree", Mathematical Social Sciences, 3, 389-396.

[15] Fleurbaey, M. and F. Maniquet. 1997. "Implementability and Horizontal equity Implies No-Envy", Econometrica, 65, 1215-1219.

[16] Gibbard, A. 1973. "Manipulation of Voting Schemes : A General result", Econometrica, $41,587-602$.

[17] Gibbard, A. 1977. "Manipulation of Voting Schemes that Mix Voting with Chance", Econometrica, 45, 665-681.

[18] Green, J. and J.J. Laffont. 1979. "On Coalition Incentive Compatibility", Review of Economic Studies, 46, 243-254. 
[19] Jackson, M.O. 2001. "A Crash Course in Implementation Theory", Social Choice and Welfare, 18, 655-708.

[20] Kim, K.H. and F.W. Roush. 1980. "Special Domains and Nonmanipulability", Mathematical Social Sciences, 1, 85-92.

[21] Laffond, G. 1980. "Révélation des préférences et Utilités Unimodales", Unpublished Dissertation, Laboratoire d'Econométrie, Conservatoire National des Arts et Métiers.

[22] Laffont, J.J. and D. Martimort. 1997. "Collusion under Asymmetric Information", Econometrica, 65, 875-911.

[23] Laffont, J.J. and E. Maskin. 1980. "A Differential Approach to Dominant Strategy Mechanisms", Econometrica, 48, 1507-1520.

[24] Le Breton, M. and A. Sen. 1999. "Separable Preferences, Decomposability and StrategyProofness", Econometrica, 67, 605-628.

[25] Le Breton, M. and J.A. Weymark. 1999. "Strategy-Proof Social Choice Functions with Continuous Separable Preferences", Journal of Mathematical Economics, 32, 47-85.

[26] Maskin, E. 1979. "Implementation and Strong Nash Equilibrium", Chapter 23 in Agregation and Revelation of Preferences, J.J. Laffont (Eds), Amsterdam, North-Holland.

[27] Moulin, H. 1980. "On Strategy-Proofness and Single Peakedness", Public Choice, 35, $437-455$.

[28] Moulin, H. 1993. "On the Fair and Coalition Strategy-Proof Allocation of Private Goods", in Frontiers in Game Theory, K. Binmore, A. Kirman and P. Tani (Eds), Cambridge : MIT University Press, 151-163.

[29] Moulin, H. 1994. "Serial Cost Sharing of Excludable Public Goods", Review of Economic Studies, 61, 305-325.

[30] Moulin, H. 1999. "Incremental Cost Sharing : Characterization by Group Strategyproofness", Social Choice and Welfare, 16, 279-320.

[31] Muller, E. and M. Satterthwaite. 1977. "On the Equivalence of Strong Positive Association and Strategy-Proofness", Journal of Economic Theory, 14, 412-418.

[32] Rockafellar, T.R. 1970. Convex Analysis, Princeton : Princeton University Press. 
[33] Satterthwaite, M. 1975. "Strategy-Proofness and Arrow's Conditions : Existence and Correspondence Theorems for Voting Procedures and Social Welfare Functions", Journal of Economic Theory, 10, 187-217.

[34] Satterthwaite, M. and H. Sonnenschein. 1981. "Strategyproof Allocation Mechanisms at Differentiable Points", Review of Economic Studies, 48, 587-597.

[35] Schummer, J. and R.V. Vohra. 2002. "Strategy-Proof Location on a Network", Journal of Economic Theory, 104, 405-428.

[36] Sprumont, Y. 1995. "Strategy-Proof Collective Choice in Economic and Political Environments", Canadian Journal of Economics, 28, 68-107.

[37] Tanaka, Y. 2002. "Strategy-Proofness of Social Choice Functions and Non-Negative Association Property with Continuous Preferences", Economics Bulletin, 4, 1-7.

[38] Zhou, L. 1991. "Impossibility of Strategy-Proof Mechanisms in Economies with Pure Public Goods", Review of Economic Studies, 58, 107-119. 